\section{Pädiater kooperieren mit} Impfstoffversender

PädNetzS, das Netzwerk baden-württemberger Pädiater, setzt die Kooperation mit dem bundesweiten Impfstoffversender a1.direkt fort. Vorstandsmitglied Ulrich Kuhn betont, "dass man angesichts düsterer Aussichten am Reformhimmel auf die tatkräftige Schützenhilfe von a1.direkt gerne zurückgreift und die Zusammenarbeit 2011 intensiviert“. Da Pädiater als impfaktive Fachärzte eine zentrale Kundengruppe von a1.direkt sind, lag es nahe, dem PädNetzS nicht nur beim Impfstoffversand interessante Serviceleistungen anzubieten, sondern es auch hinsichtlich der gesundheitspolitischen Entwicklung zu unterstützen.

Nach Informationen von a1.direkt, Lorsch

\section{Neuer Impfstoff gegen}

Meningokokken B

Novartis hat bei der European Medicines Agency (EMA) die Zulassung von Bexsero ${ }^{\circledR}$ beantragt, eines Mehrkomponenten-Impfstoffs gegen Meningokokken-Stämme der Serogruppe B. Nach Zulassung wird Bexsero ${ }^{\circledR}$ der erste Impfstoff zur Prävention von Meningokokken-B-Erkrankungen in der EU und im Europäischen Wirtschaftsraum sein, der eine breite Stammabdeckung bietet.

Nach Informationen von Novartis Vaccines and Diagnostics, Marburg

\section{Neue Fixkombination für Asthma}

Vor kurzem wurden aktuelle Studiendaten zu einer neuen Fixkombination aus dem inhalativen Kortikosteroid Fluticasonproprionat (FP) und dem langwirksamen $\beta 2$-Agonisten Formoterolfumarat (FORM) veröffentlicht. Sie zeigen, dass FP/FORM bei der Behandlung von Asthma genauso effektiv ist wie das zu den europäischen Marktführern zählende FP/Salmeterolxinofoat, die Wirkung jedoch rascher eintritt. Darüber hinaus konnte belegt werden, dass die FP/FORM-Kombination eine effektivere Kontrolle der Asthmasymptome ermöglicht als FP allein. Die Daten belegen das gute Wirksamkeitsprofil der FP/FORMKombination und unterstreichen ihr Potenzial, eine alternative Behandlungsoption für Menschen mit Asthma zu werden. Die Zulassung für die Fixkombination wurde bereits in der ersten Jahreshälfte 2010 in mehreren europäischen Mitgliedsstaaten beantragt.

Nach Informationen von Mundipharma,

Limburg

\title{
Epilepsie: Therapiestrategien für die ganz Kleinen
}

- Bei frühkindlichen idiopathischen fokalen Epilepsien ist sehr streng zu prüfen, ob eine Pharmakotherapie indiziert ist. Etwa 25\% der betroffenen kleinen Patienten mit idiopathischen fokalen Epilepsien, die häufig in der frühen Kindheit erstmals auftreten und die oft eine lebenslange Therapie erfordern, gelten als „schwer behandelbar". Bei ihnen lässt sich eine Anfallsfreiheit mit den wenigen zur Verfügung stehenden Präparaten nicht oder manchmal nur unter Inkaufnahme gravierender Nebenwirkungen bzw. Wechselwirkungen erreichen. Es fehlten bislang entsprechende Zulassungen für neuere, besser verträgliche Antiepileptika. Die unlängst erteilte Zulassungserweiterung für Levetiracetam (Keppra®) zur Zusatzbehandlung fokaler Anfälle bei Säuglingen und Kleinkindern ab einem Monat erweitert das Spektrum der Therapieoptionen. Vorteilhaft ist das pharmakologische Profil von Levetiracetam: Die fehlende Enzyminduktion, keine relevanten Interaktionen mit anderen Medikamenten, keine Verstoffwechslung über die Leber und die lineare Dosis-Wirkungs-Beziehung sind besonders vorteilhaft für die Therapie von Säuglingen und Kindern.
Ist eine medikamentöse Therapie erforderlich, empfiehlt sich laut PD Dr. Thomas Bast aus Kork-Kehl zunächst der Einsatz von Sultiam, beginnend mit $4 \mathrm{mg} / \mathrm{kg}$ KG. Die Dosis kann auf 6 oder $8 \mathrm{mg} / \mathrm{kg}$ gesteigert werden. Wird diese Therapie nicht vertragen oder ist sie nicht wirksam, sei Levetiracetam ( $30 \mathrm{mg} /$ $\mathrm{kg}$, gegebenenfalls auf 45 oder $60 \mathrm{mg} / \mathrm{kg}$ steigern) die nächste Option. Bei Unverträglichkeit oder unzureichender Wirksamkeit stellten Oxcarbazepin und Valproat Alternativen dar. Als Reservemedikamente kämen unter anderem Topiramat und Clobazam infrage. Bei atypischen klinischen und EEG-Zeichen sollten Carbamazepin, Oxcarbazepin, Lamotrigin, Phenytoin und Phenobarbital nach Auffassung Basts nicht eingesetzt werden. Droht eine Entwicklungsgefährdung der Säuglinge, sollten spätestens an dritter Stelle Steroide zum Einsatz kommen.

Dr. Gunter Freesef

Satelliten-Symposium „Frühkindliche idiopathische fokale Epilepsien: ein Update" anlässlich der 83. DGN-Jahrestagung/Neurowoche. Mannheim, 24. September 2010. Veranstalter: UCB Pharma, Monheim

\section{Mützen und Kopfkissen übertragen keine Kopfläuse}

— Kopfläuse werden durch Kopf-zu-KopfKontakt übertragen. Die indirekte Übertragung, z. B. durch Mützen oder Bettwäsche, ist kaum relevant. Das zeigt eine aktuelle Übersichtsarbeit von Canyon et al. So überleben Läuse auf leblosen Gegenständen je nach Umgebungsbedingungen nur einige Stunden. Zudem wird vermutet, dass Läuse, die den Kopf verlassen und sich auf Gegenständen niederlassen, nicht mehr lange lebensfähig sind. Auch die Überlebensfähigkeit von Läuseeiern auf Kämmen oder Bettbezügen ist gering. Die Ergebnisse aussagefähiger Feldstudien gehen in die gleiche Richtung. Dass Läuse mittels Mützen, Teppichen, Bürsten oder Bettwäsche übertragen werden, erwies sich auch hier als untergeordnetes Risiko. Die Übertragungsrate vom Kopf auf das Kopfkissen betrug lediglich 4,2\%. Bezogen auf die Gesamtpopulation der auf den Köpfen gezählten Läu- se verließen nur 0,1\% der Läuse den Kopf und ließen sich auf dem Kopfkissen nieder. Bei Kopflausbefall sollte deshalb mit Blick auf die Therapie und die Prävention die Entfernung der Läuse auf dem Kopf im Vordergrund stehen. Experten fordern eine hohe Effektivität von Pedikuloziden bei gleichzeitig guter Verträglichkeit. Erfüllt werden können diese Erwartungen von NYDA ${ }^{\oplus}$, einem Pedikulozid auf der Basis von Dimeticon. Es enthält zwei Silikonöle unterschiedlicher Viskosität und Flüchtigkeit, die tief in die Atemöffnungen der Läuse und ihrer Entwicklungsstadien eindringen und sie verschließen. Das Präparat steht inzwischen auch als NYDA ${ }^{\oplus}$ sensitiv ohne Duft- und Zusatzstoffe zur Verfügung - mit ebenso hoher Wirkung!

Nach Informationen von Pohl Boskamp, Hohenlockstedt 